\title{
PERCEPÇÕES E PRÁTICAS DE PROFISSIONAIS DE SAÚDE NO CUIDADO A PESSOAS IDOSAS HOSPITALIZADAS
}

\author{
PERCEPTIONS AND PRACTICES OF HEALTH CARE \\ IN ELDERLY HISPITALIZED
}

\section{PERCEPCIONES Y PRÁCTICAS DE CUIDADO DE SALUD EN UN ANCIANO HOSPITALIZADO}

\author{
Luciana Araújo dos Reis*, Edméia Campos Meira**, Andréa dos Santos Souza***, \\ Camila Calhau Andrade****
}

\begin{abstract}
Autor correspondente: Luciana Araújo dos Reis - lucianauesb@yahoo.com.br
*Fisioterapeuta, Mestre e Doutora em Ciências da Saúde/UFRN, Estágio Pós-doutoral em Saúde Coletiva/UFBA/ISC.

Docente Adjunta da Universidade Estadual do Sudoeste da Bahia e Docente Titular da Faculdade Independente do Nordeste, **Enfermeira. Mestre em Enfermagem. Docente Adjunta do Departamento de Saúde da Universidade Estadual do Sudoeste da Bahia/UESB, ${ }^{* * *}$ Enfermeira. Mestre e doutoranda em Enfermagem/UFBA. Docente Adjunta do Departamento de Saúde da UESB, ${ }^{* * * *}$ Enfermeira. Universidade Estadual do Sudoeste da Bahia/UESB.
\end{abstract}

\section{RESUMO}

Objetivo: conhecer como os profissionais de saúde de uma rede hospitalar pública lidam com o processo de cuidar do idoso hospitalizado, considerando legislações relacionadas à saúde da pessoa idosa. Metodologia: trata-se de um estudo qualitativo, descritivo, exploratório, realizado em um hospital público do interior da Bahia, tendo como amostra 14 profissionais de saúde atuantes nas unidades de Emergência, Terapia Intensiva e Clínicas Médica e Neurológica. Foram utilizados um formulário de identificação sociodemográfica e um roteiro para entrevista semiestruturada. Resultados: após coleta e análise de dados três (03) categorias emergiram, a saber: Representatividade da População Idosa no Serviço Hospitalar, Políticas Públicas de Saúde para a Pessoa Idosa: Percepção dos Profissionais e Assistência e Violação de Direitos da Pessoa Idosa Hospitalizada. Conclusão: Deparamo-nos com profissionais que não conhecem as leis de amparo à pessoa idosa, mas que reconhecem a sua importância e percebem as deficiências para a sua aplicabilidade no dia-a-dia de serviços.

Palavras-chave: Profissionais de saúde. Hospitalização. Saúde do idoso. 


\begin{abstract}
Objective: to understand how healthcare professionals of a public hospital network deal with the process of care for hospitalized elderly considering legislation related to health of the elderly. Methodology: this is a qualitative, descriptive, exploratory, performed in a public hospital in the interior of Bahia, and a sample of 14 health professionals working in emergency units, Intensive Care and Clinical Medicine and Neurology. We used a form of identification and a roadmap for sociodemographic semistructured interview. Results: after collecting and analyzing data, 03 categories emerged, namely: Representation of the Elderly in Hospital Service, Public Health Policies for the Elderly: Perceptions of Professional and Service and Violation of Rights of Hospitalized Elderly. Conclusion: we come across professionals who do not know the laws of protection to the elderly, but they recognize its importance and applicability to realize the shortcomings of these day-to-day services.
\end{abstract}

Keywords: Health. Hospitalization. Health of the elderly.

\title{
RESUMEN
}

Objetivo: comprender cómo los profesionales de la salud en un hospital de la red pública trato con el proceso de atención a ancianos hospitalizados considerando legislación relacionada con la salud de las personas mayores. Metodología: se trata de un estudio cualitativo, descriptivo, exploratorio, realizado en un hospital público del interior de Bahía, y una muestra de 14 profesionales de la salud que trabajan en las unidades de urgencias, cuidados intensivos y medicina clínica y Neurología. Se utilizó un formulario de identificación y una hoja de ruta para la entrevista semiestructurada sociodemográfica. Resultados: después de recoger y analizar datos, surgieron O3 categorías, a saber: Representación de las Personas Mayores en el Servicio de Hospital, Políticas Públicas de Salud para la Tercera Edad: Percepciones de los profesionales y de servicio y Violación de los Derechos de los ancianos hospitalizados. Conclusión: nos encontramos con profesionales que no conocen las leyes de protección a los ancianos, pero reconocen su importancia y aplicabilidad a darse cuenta de las deficiencias de los servicios del día a día.

Palabras clave: Salud. Hospitalización. Salud de las personas mayores.

\section{INTRODUÇÃO}

Em vários países, as populações estão envelhecendo. Estudos mostram que o número de pessoas idosas cresce em ritmo maior do que o número de pessoas que nascem, acarretando um conjunto de situações que modificam a estrutura de gastos dos países em uma série de áreas importantes como saúde e previdência social. ${ }^{(1-2)}$ Essa mudança demográfica repercutirá na dinâmica das organi- zações de saúde sendo necessária, em especial, a reformulação dos serviços de saúde para o atendimento a esse novo perfil epidemiográfico.

Nesta população, as internações hospitalares são mais frequentes e o tempo de ocupação do leito é maior entre os idosos quando comparado a outras faixas etárias. Isto corrobora a necessidade de análises periódicas de como se compõem a assistência 
e $o$ atendimento dos idosos nos serviços públicos de saúde, assim como se dá a verificação de ações políticas que protejam esta parte da população. ${ }^{(3-4)}$

De fato, entende-se que o profissional de saúde integra as condições de espectador e protagonista na realização das ações de atendimento e assistência ao idoso e estas devem ser pautadas em um compromisso que ultrapassa a dimensão profissional, incluindo a ética nas relações pessoais, possibilitando às pessoas idosas uma melhor qualidade de vida. É necessário, portanto, que todos os profissionais envolvidos no processo de assistência à saúde do idoso estejam conscientes e engajados na execução das políticas de saúde para a pessoa idosa e busquem atender as necessidades biopsicossociais do indivíduo em diversos níveis de complexidade.

Sendo assim, novas competências se impõem ao trabalho em saúde, de forma desafiadora, além da necessidade de reformulação dos serviços de saúde para o atendimento das demandas emergentes oriundas desse novo perfil epidemiológico do país. (5-6) Deste modo, percebe-se que o idoso requer um cuidado integral frente às demandas do processo de envelhecimento. A integralidade na assistência à saúde abrange as ações de promoção, prevenção, tratamento, reabilitação e garantia a todos os níveis de complexidade do Sistema de Saúde. ${ }^{(7-9)}$

Os direitos fundamentais do idoso, com destaque para a assistência em saúde, são assegurados pela legislação, no entanto, em muitos momentos, parece haver um desencontro entre as conquistas obtidas no plano formal e a prática cotidiana dos serviços de saúde com vistas a garantir os seus direitos adquiridos. ${ }^{(10-11)}$ Desta forma, o presente artigo tem por objetivo conhecer como os profissionais de saúde de um serviço hospitalar público lidam com o processo de cuidar do idoso hospitalizado, considerando legislações relacionadas à saúde da pessoa idosa.

\section{METODOLOGIA}

Trata-se de uma pesquisa de caráter descritivo/exploratório com abordagem qualitativa. $\mathrm{O}$ caráter descritivo dessa pesquisa se justifica pela proposta de estudo do nível de atendimento de um órgão público a uma comunidade, com a descrição das características de determinados fenômenos (atendimento e assistência à pessoa idosa hospitalizada) e o estabelecimento de relações entre variáveis.

O estudo foi realizado em um hospital público no interior do estado da Bahia; o hospital caracteriza-se como o único regional da rede SUS, sendo referência para aproximadamente 30 municípios, e campo de estágio para estudantes da área de saúde de uma Universidade, uma Faculdade e de Escolas Técnicas de Enfermagem do município.

Neste contexto, catorze (14) profissionais de saúde, lotados em setores desse hospital - Pronto-Socorro, Clínica Médica, Clínica Neurológica e Unidade de Terapia Intensiva (UTI) -, cujo fluxo de atendimento e assistência a pessoas idosas é maior, aceitaram livremente participar e contribuir com o presente estudo.

Para a coleta de dados, foi desenvolvido e utilizado um questionário semiestruturado composto de questões discursivas que foram enunciadas pelo entrevistador após a leitura e assinatura do termo de consentimento livre e esclarecido (TCLE) pelos entrevistados. Posteriormente, utilizou-se de um gravador para registro das entrevistas e possibilidade de transcrição das falas desses profissionais, favorecendo assim a análise de dados.

O roteiro para coleta dos dados foi constituído de duas partes: a primeira contendo dados de identificação, tais como sexo, idade, formação, grau de formação, tempo de formação e a segunda parte contendo questões relacionadas à especificidade do atendimento e assistência de idosos nos serviços de saúde, sendo que todas essas questões foram formuladas a partir dos artigos e incisos do EI, da PNSPI e da PNH/SUS que basicamente se configuram como o pano de fundo desta pesquisa.

$\mathrm{Na}$ busca de atingir os objetivos do estudo, utilizamos a técnica de análise de conteúdo de Bardin ${ }^{12}$ que consiste em descobrir os núcleos de sentido que compõem uma comunicação cuja frequência ou presença signifiquem alguma coisa para o objeto 
analítico visado. ${ }^{(13)}$ Este estudo tem aprovação do Comitê de Ética em Pesquisa da UESB, através do protocolo $n^{\circ} 221 / 2008$.

\section{RESULTADOS E DISCUSSÃO}

A partir da investigação das características sociodemográficas dos profissionais verificou-se que a maioria se encontra na faixa etária de 20 a 30 anos $(n=5)$ e de 31 a 40 anos $(n=5)$; quanto ao sexo dos profissionais houve uma maior distribuição do sexo feminino $(n=12)$. Com relação à formação profissional foi considerado o quantitativo de 05 enfermeiros, 04 técnicos de enfermagem, 02 assistentes sociais, 02 fisioterapeutas e 01 psicólogo. Não houve participação do profissional médico, devido à dificuldade de disponibilidade de tais profissionais para a pesquisa.

Em se tratando do tempo de formação, temos que $n=5$ possuem menos de 05 anos de experiência profissional, seguido por $n=04$ entre 6 a 10 anos, $\mathrm{n}=03$ com 11 a 15 anos, $\mathrm{n}=4$ entre 21 a 25 anos e $\mathrm{n}=3 \mathrm{com}$ mais de 26 anos de experiência profissional. Ainda conhecendo como ocorre o processo de trabalho, investigamos que $n=5$ profissionais possuem 02 vínculos empregatícios e $n=2$ possuem 03 vínculos.

A partir das informações fornecidas por estes profissionais, sujeitos do estudo, emergiam os seguintes núcleos temáticos e subcategorias:

Quadro 1 - Distribuição dos Núcleos temáticos e respectivas subcategorias relativas ao Atendimento prestado à pessoa idosa nos serviços de saúde hospitalar, segundo relato dos profissionais de saúde, Jequié-BA, 2013.

\begin{tabular}{|c|c|}
\hline NÚCLEOS TEMÁTICOS & SUBCATEGORIAS/NÚCLEOS \\
\hline \multirow{2}{*}{$\begin{array}{l}\text { Representatividade da População Idosa } \\
\text { no Serviço Hospitalar. }\end{array}$} & $\begin{array}{l}\text { Subcategoria } 1.1 \text { - Demografia e Epidemiologia da População } \\
\text { idosa. }\end{array}$ \\
\hline & Subcategoria 1.2 - A Gestão e Efetividade da Atenção Básica. \\
\hline \multirow{2}{*}{$\begin{array}{l}\text { Concepção de Profissionais de Saúde } \\
\text { sobre Políticas Públicas de Saúde para a } \\
\text { pessoa idosa. }\end{array}$} & $\begin{array}{l}\text { Subcategoria } 2.1 \text { - Conhecimento das Políticas Públicas de } \\
\text { Saúde. }\end{array}$ \\
\hline & $\begin{array}{l}\text { Subcategoria } 2.2 \text { - Importância atribuída às Políticas Públicas } \\
\text { de Saúde e Efetivação }\end{array}$ \\
\hline \multirow{2}{*}{$\begin{array}{l}\text { Assistência e Violação de Direitos da } \\
\text { Pessoa Idosa Hospitalizada. }\end{array}$} & $\begin{array}{l}\text { Subcategoria } 3.1 \text { - Percepções para o Crime na Assistência à } \\
\text { Pessoa Idosa }\end{array}$ \\
\hline & Subcategoria 3.2 - Violação de Direitos: Denúncias e Causas \\
\hline
\end{tabular}

Fonte: Dados da pesquisa

\section{CATEGORIA 1 - REPRESENTATIVIDADE DA POPULAÇÃO IDOSA NO SERVIÇO HOSPITALAR}

A partir dos relatos dos profissionais entrevistados, pudemos perceber que todos consideram significativa a parcela de pessoas idosas hospitalizadas, nos setores em que trabalham: Pronto-Socorro, Clínicas Médica e Neurológica e Unidade de Terapia Intensiva:

[...] na emergência a gente tem. Muitos idosos mesmo. Bastante representativa. Aqui (Clínica 
médica) a gente trabalha em torno de $80 \%$ dos pacientes internados com a faixa etária acima de 70 anos. Com certeza. Hoje, por exemplo, de dez pacientes na UTI, seis pacientes são idosos, mais de $50 \%$ e normalmente é essa a faixa que tem se mantido [...].

Estes resultados, obtidos a partir das vivências e práticas cotidianas destes profissionais, corroboram com outros estudos que discorrem a respeito da prevalência da população idosa nas autorizações de internações hospitalares emitidas pelo SUS. ${ }^{(10)}$ A partir desta constatação, podemos questionar quais são os motivos desta alta frequência de hospitalizações de idosos.

SUBCATEGORIA 1.1 - Demografia e Epidemiologia da População Idosa

Do total dos profissionais entrevistados, $n=4$ relacionaram a demanda de hospitalizações diretamente ao crescimento de idosos, em números relativos e absolutos no total da população. $\mathrm{Na}$ percepção dos profissionais, à medida que cresce o número de pessoas com mais de 60 anos há uma repercussão disto nos principais segmentos sociais, incluindo o serviço hospitalar público:

[...] é o envelhecimento, a qualidade de vida que faz com que a população tenha mais tempo de vida e isso vem crescendo, né, a nivel nacional, então, nós tínhamos uma população jovem no Brasil e ela verm alterando. Nossa realidade hoje é de uma população que tá envelhecendo, né, ela é muito grande, então eu acredito que se você entrar no hospital hoje, a gente vai ter uma média de 40 a $50 \%$ de idoso. Primeiro, Jequié é uma cidade longeva, tem muito idoso em Jequié [...].

Estas percepções confirmam o contexto demográfico em que esforços foram feitos para o aumento da expectativa de vida e isto se tornou pos- sível. Entretanto, vários estudos têm comprovado que associada a esta mudança demográfica que vem ocorrendo não só na cidade de Jequié, mas no cenário da população brasileira, de maneira geral, ocorrem paralelamente alterações epidemiológicas no quadro de morbimortalidade da população. As doenças agudas e de origem infecciosa têm tido incidências reduzidas. Em contrapartida, tem ocorrido um aumento significativo da prevalência de doenças crônico-degenerativas. ${ }^{(14)}$

Neste estudo, $\mathrm{n}=8$ sujeitos citaram as doenças crônico-degenerativas e as suas complicações como fatores das elevadas taxas de hospitalizações de idosos:

[...] o fato mesmo, eu acho que, pressão alta [...] é muita, muita gente hipertensiva, a maioria dos idosos que dá entrada aqui no Pronto Socorro é de AVC ou infarto. O idoso é internado por diabetes descompensada, hipertensão, pé diabético. [...] a gente recebe muitos pacientes idosos com AVC, DPOC, pneumonia. A maioria dos nossos pacientes são pacientes já crônicos, idosos de AVC, sequelados.

\section{SUBCATEGORIA 1.2 - Efetividade da}

\section{Atenção Básica}

Diversos fatores podem motivar os idosos a procurarem os serviços de saúde hospitalares, dentre eles a fragilização no processo de envelhecimento e a não resolutividade dos problemas de saúde de natureza crônica nos níveis de assistência primário e secundário. Na transição epidemiológica brasileira ocorrem incapacidades resultantes do não controle de fatores de risco preveníveis. ${ }^{(15)}$

Alguns dos profissionais entrevistados apontaram este fato como responsável pela alta demanda de idosos no hospital:

[...] e em relação à atenção básica, que eu não vejo um atendimento assim, eu não sei se eu posso tá julgando por não tá lá, mas o que eu 
observo é, os idosos, AVC, cardiopatas, quando a gente começa a pegar a anamnese, a gente descobre que eles não tomam a medicação certa, tomam quando dá, quando lembram... então eu vejo uma forma de cuidado precária, tanto pelo cuidador como pelo pessoal da atenção. A gente vê aqui na médica, o idoso é internado por diabetes descompensada, hipertensão, pé diabético, são doenças que poderiam ter sido tratadas antes no Centro de Saúde, numa Unidade Básica, como nós não temos esse apoio da Unidade Básica, essas doenças tornam-se crônicas e eles tem que vir pra o Prado Valadares já num estado bem avançado que poderia ter sido resolvido antes e não foi[...].

O SUS apresenta como porta de entrada no sistema a Atenção Básica à Saúde (ABS), nível em que aproximadamente $80 \%$ das necessidades de saúde de uma comunidade adstrita devem ser abordadas de maneira resolutiva. ${ }^{(14)}$ Nesta perspectiva, para o cuidado dos idosos é necessária a oferta de serviços que possibilitem o acesso e acolhimento destes sujeitos, de modo a respeitar limitações que proporções relevantes de idosos apresentam. ${ }^{(14)}$

\section{CATEGORIA 2 - CONCEPÇÃO DE PROFISSIONAIS SOBRE POLÍTICAS PÚBLICAS PARA A PESSOA IDOSA}

\section{SUBCATEGORIA 2.1 - Conhecimento} das políticas públicas de saúde

Apenas $n=4$ dos sujeitos da pesquisa afirmam conhecer as políticas públicas de saúde voltadas para a pessoa idosa. Outros $n=4$ afirmam já terem ouvido falar, mas não se lembram quais são e $n=6$ afirmaram, simplesmente, não conhecer nada a respeito.

Desta forma, sentimos falta de investimentos em pesquisas e incentivos públicos e privados para dinamizar e otimizar as políticas para esse segmento da sociedade. O Estado deve, portanto, estar atento às próprias políticas públicas de saúde e priorizar a divulgação para que os profissionais da saúde sejam capacitados e estejam cientes das peculiaridades que envolvem o agir em saúde, frente às necessidades de um idoso, reconhecendo-se como agente de responsabilidade legal. Sobre isso um profissional comentou:

[...] Elas (políticas de saúde para a pessoa idosa) têm que ser conhecidas pelo trabalhador da saúde e não são ainda. Existe uma distância entre a elaboração, regulamentação da política e a execução dela, então a gente precisava primeiro divulgar para os trabalhadores $e$ depois implementar, então eu acho que essa é a primeira dificuldade, como várias leis no Brasil, elas existem já no papel mas na hora de efetiva, falta divulgação[...].

Portanto, uma das alternativas mais importantes para assegurar a autonomia e independência do idoso, como também o envelhecer saudável, sem dúvida são as ações educativas de maneira extensiva, abrangendo não só esta parcela da sociedade mas também o pessoal da saúde. Estado, profissionais da saúde, idoso e sociedade, em geral, são todos corresponsáveis por esse processo.

\section{SUBCATEGORIA 2.2 - Importância}

atribuída às Políticas Públicas de Saúde voltadas para pessoa Idosa e Efetivação

Apesar de não conhecerem as leis de amparo à pessoa idosa em vigência, os profissionais entrevistados reconhecem a importância da formulação de políticas públicas de Saúde voltadas para pessoa Idosa e Efetivação. Além disso, percebem deficiências na aplicabilidade dessas políticas no dia-a-dia de serviços:

[...] as Políticas Públicas são muito bem elaboradas, né, o SUS é muito bem estruturado, existe uma parte teórica que se fosse colocada em 
prática, como tá ali, teríamos problemas, cla ro, mas seriam bem minimizados, então assim, elas são importantíssimas, são avanços, né, se nós imaginarmos que anos atrás, não existiam [...] o problema está na efetivação delas. Ah, eu acho de suma importância, eu acho que tem que sair um pouco do papel e ir pra prática. Eu acho de grande relevância, principalmente que nós sabemos que o Brasil está envelhecendo. Então realmente tem que se repensar essas políticas, tem que fazer com que elas realmente aconteçam de maneira eficaz porque nós seremos um país idoso, então a gente tem que se preparar pra essa fase[...]

O Art. $9^{\circ}$ do Estatuto do Idoso dispõe que é obrigação do Estado garantir à pessoa idosa a proteção à vida e a saúde, mediante políticas sociais públicas que permitam um envelhecimento saudável e em condições de dignidade de maneira efetiva. Entretanto, neste estudo, $n=11$ profissionais afirmaram que há dificuldades para a efetivação das Políticas. Dentre eles, $n=8$ referiram-se à pesada jornada de trabalho aliado ao pequeno número de profissionais como dificultadores diretos desse processo:

[...] a sobrecarga humana é muito grande em um ambiente de estrutura pequena, a gente acaba não atendendo pela grande demanda que tem, a gente tem uma unidade de emergência que tem 16 leitos somente de observação, e a gente chega dia que tem mais de 43 pacientes internados, então não tem como a gente dá esse atendimento [...] a gente tenta da melhor maneira cuidar, dar atenção adequada, mas você sabe, pelo pouco número de funcionários, a gente não dispõe daquela atenção que eles deveriam ter, entendeu, mas a gente tenta o melhor aqui, fazer o melhor. Assim, é meio complicado pela própria demanda. A gente tem um hospital, um único hospital da cidade que é referencia no trauma. A gente tem um número de acidentes muito grande e assim, algumas coisas ficam sem serem feitas, mas não por conta do não conhecimento, né, mas sim pela própria demanda, né, quem são mais graves acaba tendo atendimento prioritário [...].

\section{CATEGORIA 3 - CONCEPÇÃO DE PROFISSIONAIS DE SAÚDE SOBRE VIOLAÇÃO DOS DIREITOS DA PESSOA IDOSA HOSPITALIZADA}

Alguns idosos, na busca por atendimento em um serviço de saúde, podem se tornar vítimas de várias ações que violentam e violam seus direitos previstos em legislação. Rituais de agressões, violências e maus tratos que se desenrolam contra a pessoa idosa expressam-se na força, na palavra, no silêncio, na omissão, na posse. Falar em violência, agressões e maus tratos significa falar de traumas de natureza psicológica, emocional, moral e física. (17)

SUBCATEGORIA 3.1 - Percepções de profissionais para "crime" durante o processo de cuidar

Aos profissionais de saúde entrevistados, foi-lhes questionado sobre o que definem como "crime" no contexto durante a assistência à pessoa idosa hospitalizada. As seguintes respostas foram selecionadas para análise e discussão:

[...] o preconceito, porque às vezes a gente negligência uma dor, fala assim: veio pra cá, por causa de uma dor, o idoso sente dor mesmo... então eu acho que isso é um crime porque a gente não tá pra pesar e nem dosar a dor de ninguém, você sente muito quando você vê um idoso em um corredor, né, um idoso que tá ali fragilizado, ele fica sentando em um lugar duro, que não tem conforto nenhum e que isso acaba acarretando pra ele outros problemas. A negligência ao idoso eu acho que é um crime... a qualquer pessoa, na verdade, que precise de 
ajuda ou de cuidado. Maus tratos, que às vezes a gente vê muito, a gente vê até de acompanhante. Falta de acompanhamento médico. Porque muitas vezes o que a gente vê aqui, que os médicos passam, alguns, né, não tem aquela visão mais detalhada, fica muito no superficial e aí, problemas que deveriam ser mais aprofundados não são. Omissão, a omissão de socorro a ele, a omissão a informação. Principalmente, $o$ desrespeito ao idoso [...].

No Brasil, pesquisas sobre a violência contra idosos apontam para uma cultura negligente que se manifesta nas instituições sociais e de saúde, observada nas relações de despersonalização, de destituição de poder e vontade, bem como na omissão de cuidados específicos. ${ }^{(13)}$

Nas falas dos profissionais encontramos referências à negligência no atendimento, omissão e maus tratos físicos como sinônimos de crime. O Estatuto do Idoso apresenta vários atos que podem ser considerados delitos graves e que conferem com os citados pelos profissionais.

SUBCATEGORIA 3.2 - Violação de direitos: Denúncias e Causas

Ao serem questionados se já presenciaram algum tipo de tratamento desumano, violento, vexatório ou constrangedor contra idosos hospitalizados, $57 \%$ dos profissionais responderam que sim:

[...] constrangedor, inúmeros. Por exemplo, eu considero constrangedor a gente ver num corredor de emergência, um paciente todo evacuado e não ter uma privacidade pra gente realizar essa higiene nele, a gente precisa fazer aquele biombo humano, juntar 2, 3 pessoas, pra tentar uma forma de realizar aquela higiene no meio de um corredor, então pra mim, isso já é um tratamento desumano. Rotineiramente a gente vê pela pressa que os profissionais têm, não dá atenção, o idoso pergunta, ele não responde, ou às vezes fica muito tempo de xixi ou tá sentin- do dor, reclama e o profissional não tem tempo pra dar assistência naquele momento. O que me angustia é essa questão do corredor de ficar exposto, às vezes ele tá ali de fralda, de sonda, sem roupa, ele tá com uma ferida no pé no odor fétido e todo mundo rejeita, é um mau trato, ninguém chega perto por causa daquele odor, aí isso eu já vi, é constrangedor [...].

Todas as situações relatadas são indiscutivelmente violação dos direitos da Pessoa Idosa enquanto cidadã de respeito e dignidade. O Art. $4^{\circ}$ do Estatuto do Idoso traz que nenhum idoso será objeto de qualquer tipo de negligência, discriminação, violência, crueldade ou opressão e todo atentado aos seus direitos, por ação ou omissão, deverá ser punido na forma da lei. Além disso, é dever de todos prevenir a ameaça ou violação dos direitos do idoso.

$\mathrm{Na}$ verdade, as hipóteses que tentam explicar os fatores relacionados à violência contra pessoas idosas nas instituições de saúde são de natureza multifatorial e complexa, e guardam estreita relação entre si. ${ }^{(17)}$

Podem estar relacionadas às condições de trabalho nos serviços públicos de saúde, a formação profissional e aos aspectos subjetivos como comportamento individual, personalidade, sensibilidade para com o sofrimento do outro, moral, valores e ética; relacionam-se também a fatores macrossociais como: a precariedade da assistência às pessoas de todos os grupos etários, a cultura da violência, as representações sociais da velhice, o capitalismo, o paradigma biomédico de cuidado, dominação e conflitos intergeracionais. A questão cultural foi discutida por um profissional:

[...] a gente sabe que é uma questão cultural, infelizmente nosso país, está no ocidente, ele não foi preparado. Não é como o oriente, que tem todo um olhar de valorização do idoso. Então por uma questão cultural, as pessoas não valorizam esse idoso, 
não observa ele como uma fonte de riqueza de conhecimento. (P11)

Diante do exposto, observa-se que a violência institucional também pode ser analisada como uma conduta cultural cujas relações de desvalorização são aprendidas na convivência com idosos da família, comunidade e são reproduzidas na instituição.

$\mathrm{Na}$ concepção da mesma profissional de saúde, atitudes discriminatórias em relação às pessoas idosas tendem a reduzir à medida que também vamos envelhecendo e desmistificando os estereótipos negativos construídos, como observado no relato a seguir:

[...] então assim, a gente passa a ter um olhar, no momento em que o tempo vai passando, vai começando a ter um olhar diferenciado $e$ isso seria interessante se acontecesse desde pequeno, porque ganharia todo mundo. Os conflitos de gerações não eram tantos. $\left(P_{11}\right)$

Na percepção de alguns profissionais investigados, a discriminação de idosos existe nos serviços de saúde aonde trabalham. Sob a perspectiva econômica são vistos como uma carga que ameaça a sustentabilidade financeira do sistema, pelo elevado custo, cujo retorno social é questionável. No aspecto assistencial são considerados dependentes e lentos, o que atrasa o andamento do serviço. Estereótipos de inutilidade e incompetência contribuem para uma crença equivocada de que já cumpriram sua missão, portanto não é preciso viver mais. Em algumas situações a idade tem sido utilizada como único critério para contraindicar uma conduta terapêutica. Mas, se perguntarmos aos idosos hospitalizados sobre os seus planos após a hospitalização é provável que listem metas surpreendentes.

Portanto, a violência institucional não é menos brutal que a violência intrafamiliar e, de igual modo, constitui uma violação dos Direitos Huma- nos, pois nutre sentimentos de culpa, solidão, dependência, inutilidade e aumenta a sensação de desamparo. A negligência, sua manifestação mais notória, infringe os direitos humanos básicos, como o da vida, da integridade pessoal, da dignidade e o impede de gozar do mais alto padrão de saúde possível.(18)

\section{CONCLUSÕES}

Os profissionais investigados expressaram vários modos de compreender o crime produzido: uma prática habitual produzida pelo outro (acompanhantes, colegas de trabalho e demais profissionais da equipe); uma conduta cultural, cujas relações de desvalorização aprendidas na convivência com idosos da família e comunidade são reproduzidas na instituição; uma consequência da deficiência de estrutura de serviço oferecida.

As manifestações mencionadas de violência contra a pessoa idosa hospitalizada foram: negligência, preconceito, discriminação, insuficiência de leitos, permanência insalubre em corredores, desassistência, atraso na realização de alguns procedimentos e exames diagnósticos, desrespeito ao período de repouso, negação do direito à privacidade e a ser ouvido por pressa, frieza e rispidez no atendimento dispensado pelos profissionais.

No que tange a violência institucional em serviços de saúde, concluímos que é um grave problema e de origem multifatorial. Várias questões fazem com que sintamos quão distante se está em garantir àqueles de mais idade os direitos fundamentais à vida, à integridade e à saúde, como a qualquer outro cidadão.

\section{REFERÊNCIAS}

1. IBGE, Instituto Brasileiro de Geografia e Estatística. Sinopse do Censo Demográfico 2010. Rio de Janeiro: 2011.

2. Souza $A C C$, et.al. Formação do enfermeiro para o cuidado: reflexões da prática 
profissional. Revista Brasileira de Enfermagem 2006;59(6):805-807.

3. Coelho Filho, JM. Modelos de serviços hospitalares para casos agudos em idosos. Revista de Saúde Pública 2000;34(6):666-71.

4. Brasil. Ministério da Saúde. O SUS de A a Z : garantindo saúde nos municípios / Ministério da Saúde, Conselho Nacional das Secretarias Municipais de Saúde. - 3. ed. - Brasília : Editora do Ministério da Saúde, 2009.

5. Rodrigues RAP, et. al. Política nacional de atenção ao idoso e a contribuição da enfermagem. Texto Contexto e Contexto em Enfermagem 2007;16(3).

6. Camacho ACLF, COELHO MJ. Políticas públicas para a saúde do idoso: revisão sistemática. Revista Brasileira de Enfermagem 2010; 63(2):279-84.

7. Barreira KS, VIEIRA LJES. O olhar da enfermagem para o idoso: revisão de literatura. Revista de Enfermagem da UERJ 2004;12(3):O110.

8. Motta LB, Aguiar AC. Novas Competências profissionais em saúde e o envelhecimento populacional brasileiro: integralidade, interdisciplinaridade e intersetorialidade. Ciência \& Saúde Coletiva 2007;12(2):363-372.

9. Brasil. Portaria $\mathrm{N}^{\circ} 2.528$, de 19 de outubro de 2006. Aprova a Política nacional de Saúde da Pessoa Idosa. Ministério da Saúde, Diário Oficial da União, Brasília 20 de Out. 2006.
1O. Gorzoni ML, Pires SL. Idosos asilados em hospitais gerais. Revista de Saúde Pública 2006;4O(6): p.1124-30.

11. Almeida MNBC. Hotelaria Hospitalar: cultura e hospitalidade no atendimento a idosos. Centro de Excelência em Turismo. Universidade de Brasília - Brasilia -DF, 2009.

12. Bardin L. Análise de Conteúdo. Lisboa, Portugal: Edições, 1997, p.42.

13. Minayo MCS, Sanches O. QuantitativoQualitativo: Oposição ou complementaridade? Cadernos de Saúde Pública 1993;9(3):239-262.

14. Camarano AA. A demografia e o envelhecimento populacional. In. Borges APA (Org.); Coimbra AMC (Org.). Envelhecimento e saúde da pessoa idosa. Rio de Janeiro, 2008.

15. Lima-Costa MFF. et al, Desigualdade social e saúde entre idosos brasileiros: um estudo baseado na pesquisa Nacional por Amostra de Domicilios. Cadernos de Saúde Pública 2003; 19(3):745-757.

16. Piccini, RX et al. Necessidades de saúde comuns aos idosos: efetividade na oferta e utilização em atenção básica à saúde. Ciência \& Saúde Coletiva 2006,3(11):657-667.

17. Menezes MR. Violência contra ldosos: é preciso se importar! In: Berzins MV, Malagutti W. (orgs). Rompendo o silêncio: faces da violência na velhice. São Paulo: Martinari, p. 27-58, 2010.

18. Organização Nações Unidas (ONU). Declaração Universal dos Direitos Humanos. Resolução 217 A (III) da Assembléia Geral das Nações Unidas em 10 de dezembro de 1948. 